stroke thrombectomy requiring further intracranial interventions to achieve successful recanalization. The aim of our study was to determine the prevalence of refractory thrombectomy requiring further intervention, compare their baseline characteristics, adjunctive intracranial interventions, and outcomes.

Methods We retrospectively reviewed 253 patients treated with mechanical thrombectomy for ELVO from Jan 2015 to December 20198. Refractory thrombectomy was defined as patients requiring emergent intracranial angioplasty/stenting, intraprocedural antiplatelet therapy, or patients suffering reocclusion in the same artery within one day of the procedure. Baseline characteristics and outcomes were compared between the refractory and the standard treatment groups using MannWhitney and Fisher Exact and Chi square tests. Outcomes were also compared within the refractory group undergoing different treatment modalities. Favorable outcome was defined as $\mathrm{mRS} \leq 2$ after 3 months.

Results Refractory thrombectomy was identified in 22 cases $(8.7 \%)$ with no statistically significant differences in baseline characteristics, median NIHSS score (15 vs. $17, \mathrm{P}=0.14)$, and percentage of favorable outcomes $(42.1 \%$ vs $51.9 \%, \mathrm{P}=0.48)$ versus the standard thrombectomy group, respectively. Patients in the refractory cohort had a significantly higher prevalence of diabetes $(45.5 \%$ vs. $19.9 \%, \mathrm{P}=0.013)$, while the standard group patients presented with significantly higher rates of atrial fibrillation $(47.8 \%$ vs. $18.2 \%, \mathrm{P}=0.007)$. Diabetes was the sole independent predictor of refractory thrombectomy with OR 3.436 (95\% CI: 1.190-9.524, p=0.017). Within the refractory group, 9 patients were treated with intracranial stenting (40.9\%), 7 received intra-arterial/intravenous eptifibatide infusion $(31.8 \%), 2$ patients were treated by angioplasty (9\%), and 4 developed silent re-occlusions (18\%). There were no significant differences in favorable outcomes between the standard versus refractory thrombectomy groups, whether treated with intracranial stenting or eptifibatide antiplatelet therapy $(51.9 \%$ Vs. $62.5 \%$ Vs. $14.3 \%, \mathrm{P}=0.12)$.

Conclusion Refractory thrombectomy is more common in diabetics. Emergent intracranial stenting and eptifibatide antiplatelet are equally safe and effective adjuvant treatments after stroke thrombectomy in the setting of underlying vessel wall pathology.

Disclosures R. Abdalla: None. M. Darwish: None. T. Shokuhfar: None. S. Azmi: None. D. Cantrell: None. M. Hurley: None. M. Potts: None. B. Jahromi: None. A. Shaibani: None. S. Ansari: None.

\section{E-116 CLINICAL CHARACTERISTICS, STROKE MECHANISM AND OUTCOME OF ISOLATED SYMPTOMATIC BASILAR ARTERY STENOSIS}

${ }^{1} E$ Samaniego, ${ }^{2} \mathrm{~A}$ Shaban, ${ }^{1} \mathrm{~S}$ Ortega-Gutierrez, ${ }^{3} \mathrm{D}$ Hasan, ${ }^{4} \mathrm{C}$ Derdeyn, ${ }^{5} \mathrm{~B}$ Dai, ${ }^{6} \mathrm{~J}$ Roa*, ${ }^{2} \mathrm{H}$ Adams Jr, ${ }^{2} \mathrm{E}$ Leira. ${ }^{1}$ Neurology, Neurosurgery and Radiology, University of lowa Hospitals and Clinics, lowa City, IA; ${ }^{2}$ Neurology, University of lowa Hospitals and Clinics, lowa City, IA; ${ }^{3}$ Neurosurgery, University of lowa Hospitals and Clinics, lowa City, IA; ${ }^{4}$ Radiology, University of lowa Hospitals and Clinics, lowa City, IA; ${ }^{5}$ Epidemiology and Public Health, University of lowa Hospitals and Clinics, lowa City, IA; ${ }^{6}$ Neurology and Neurosurgery, University of lowa Hospitals and Clinics, lowa City, IA

\subsection{6/neurintsurg-2019-SNIS.191}

Background While diffuse atherosclerotic disease affecting the posterior circulation has been described extensively, the prevalence, natural history and angiographic characteristics of isolated symptomatic basilar artery stenosis (ISBAS) remains unknown.

Methods We reviewed our prospectively acquired institutional database to identify patients with $\geq 50 \%$ symptomatic basilar artery (BA) stenosis without significant atherosclerotic burden in the vertebral or posterior cerebral arteries. Stroke mechanism, collateral circulation through the posterior communicating arteries, degree and length of the stenosis were analyzed. The primary outcome was time from index event to new transient ischemic attack (TIA), acute ischemic stroke (AIS) or death. Other outcome variables included modified Rankin scale (mRS) score on discharge and last follow-up.

Results Of 6369 patients with AIS/TIA, only 91 (1.43\%) had ISBAS. Seventy-three $(80.2 \%)$ patients presented with AIS and $18(19.8 \%)$ with TIA. Twenty-nine (31.9\%) were women and the median age was $66.8 \pm 13.6$ years. The mean follow-up time was 2.7 years. The most common stroke mechanism was artery-to-artery thromboembolism $(50.8 \%)$, followed by perforator occlusion $(32.3 \%)$ and flow-dependent/hypoperfusion $(16.9 \%)$. The percentage of stenosis was lower in patients who had favorable outcome compared to those with mRS 3-6 on discharge (78.3 \pm 14.3 vs $86.9 \pm 14.5, \mathrm{p}=0.007)$. Kaplan-Meier curves showed higher recurrence/death rates in patients with $\geq 80 \%$ stenosis, mid-basilar location and poor collateral circulation. Approximately $13 \%$ of ISBAS patients presented with complete BA occlusion.

Conclusion ISBAS is an uncommon $(1.5 \%)$ cause of TIAs and AIS. Males in their sixties are mostly affected and artery-toartery embolism is the most common stroke mechanism. Midbasilar location, $\geq 80 \%$ stenosis and poor collateral circulation are important factors associated with worse prognosis. Keywords: basilar artery stenosis, ischemic stroke, transient ischemic attack, intracranial atherosclerotic disease, stroke mechanism, collateral circulation, posterior circulation.

Disclosures E. Samaniego: None. A. Shaban: None. S. OrtegaGutierrez: None. D. Hasan: None. C. Derdeyn: None. B. Dai: None. J. Roa: None. H. Adams: None. E. Leira: None.

\section{E-117 OUTCOMES OF ENDOVASCULAR TREATMENT IN DIRECT VERSUS TRANSFER PATIENTS; TRANSFER STUDY: A PRELIMINARY ANALYSIS}

${ }^{1}$ A Zafar*, 'S Suriya, ${ }^{1} \mathrm{M}$ Farooqui, ${ }^{1} \mathrm{O}$ Owens, ${ }^{1} \mathrm{~A}$ Alvarado Arias, ${ }^{1} \mathrm{~A}$ Ikram, ${ }^{2} \mathrm{D}$ Sorte, ${ }^{2} \mathrm{~A}$ Carlson, ${ }^{1} \mathrm{M}$ Torbey. ${ }^{1}$ Neurology, University of New Mexico Health Sciences Center, Albuquerque, NM; ${ }^{2}$ Neurosurgery, University of New Mexico Health Sciences Center, Albuquerque, NM

10.1136/neurintsurg-2019-SNIS.192

Objectives The primary objective is to evaluate difference in outcomes after endovascular treatment (EVT) between patients directly admitted through Emergency Department (ED) and patients transferred from outside institutions.

Background Over last few years, EVT has been established as the most-effective treatment for acute ischemic stroke (AIS) patients with large vessel occlusion (LVO). In New Mexico state, University of New Mexico Hospital (UNMH) serves as the only institution providing $24 / 7$ services for EVT. In addition to transfers, patients are also admitted directly to UNMH through ED to undergo EVT. Considering the dynamics of New Mexico sate, representing various other similar states, we are conducting this study to evaluate any significant difference in the outcome of transferred patients in comparison to directly admitted patients. 
Methods This is an observational retrospective cohort study. Patients who underwent EVT for LVO from January 01, 2018 to December 31, 2018 at UNMH were included. Data was collected on secured Redcap ${ }^{\mathrm{TM}}$ database. Patients were categorized into direct admission (DA) vs transfer groups (TG). Patients who were not candidates for EVT were excluded.

Results A total of 53 patients underwent EVT. Seventeen patients were directly admitted through ED while 36 were transferred from outside hospitals. Age, gender, ethnicity and risk factor profile are reported in table 1. The two groups had similar NIHSS and GCS on presentation. The median time of arrival of patients to UNM, from last known well,

\begin{tabular}{lll}
$\begin{array}{l}\text { Abstract E-117 Table 1 } \\
\text { patients in two groups }\end{array}$ & Demographics and comorbidities of \\
\hline & $\begin{array}{l}\text { Direct Patients } \\
(\mathrm{N}=17)\end{array}$ & $\begin{array}{l}\text { Transfer Patients } \\
(\mathrm{N}=36)\end{array}$ \\
\hline Mean Age (standard deviation) & $66( \pm 13.5)$ & $71( \pm 11.6)$ \\
Gender & $8(47.0 \%)$ & $15(41.7 \%)$ \\
- Female & $9(52.9 \%)$ & $21(58.3 \%)$ \\
- Male & & \\
Ethnicity & $7(41.2 \%)$ & $19(52.8 \%)$ \\
- Caucasian & $2(11.8 \%)$ & $1(2.8 \%)$ \\
- African & $7(41.2 \%)$ & $11(30.6 \%)$ \\
- Hispanic & $1(5.9 \%)$ & $1(2.8 \%)$ \\
- Asian & $0(0 \%)$ & $1(2.7 \%)$ \\
- Native American & $0(0 \%)$ & $3(8.3 \%)$ \\
- Unknown & & \\
Medical History & $9(52.9 \%)$ & $24(66.7 \%)$ \\
- HTN & $5(29.4 \%)$ & $10(27.7 \%)$ \\
- HLD & $0(0 \%)$ & $5(13.9 \%)$ \\
- CHF & $2(11.8 \%)$ & $10(27.7 \%)$ \\
- CAD & $6(35.3 \%)$ & $12(33.3 \%)$ \\
- DM & $3(17.7 \%)$ & $16(44.4 \%)$ \\
- AF & $2(11.8 \%)$ & $4(11.1 \%)$ \\
- Previous IS & $0(0 \%)$ & $0(0 \%)$ \\
- Previous HS & $0(0 \%)$ & $12(33.3 \%)$ \\
- Tobacco & & \\
\hline
\end{tabular}

Abstract E-117 Table 2 Baseline characteristics, timings and outcomes in patients undergoing endovascular treatment

\begin{tabular}{llll}
\hline & $\begin{array}{l}\text { Direct Patients } \\
(\mathrm{N}=17)\end{array}$ & $\begin{array}{l}\text { Transfer Patients } \\
(\mathrm{N}=36)\end{array}$ & $p$-value \\
\hline Clinical baseline & 18 & 16 & 0.9 \\
- Mean NIHSS & 13 & 14 & 0.02 \\
- Mean GCS & 0 & 0 & \\
- Mean Pre-stroke mRS & 6 & 16 & \\
- Treatment with t-PA & & & 0.03 \\
Timing & 211 & 321 & 0.3 \\
- Median LKW - UNM arrival (min) & 408.5 & 373.5 & 0.3 \\
- Median LKW - Groin puncture (min) & 471 & 410 & 0.2 \\
- Median LKW- Reperfusion time (min) & 41 & 28 & 0.78 \\
-Median groin to reperfusion time (min) & & & 0.45 \\
Outcomes & 3 & 3 & \\
- Mean mRS at 3 months & 9 & 6 & \\
- Mean NIHSS at discharge & $3(17.7 \%)$ & $10(27.8 \%)$ & \\
- Hemorrhagic transformation & $4(23.5 \%)$ & $9(25 \%)$ & \\
- Mortality & & & \\
\hline
\end{tabular}

was 211 minutes in DA and 321 minutes in TG $(p=0.03)$. Median UNM arrival to groin puncture time was 121 minutes in DA and 66 minutes in TG $(\mathrm{p}=0.04)$. Median procedural time for recanalization was 41 minutes for $\mathrm{DA}$ and 28 minutes for TG. One patient had unsuccessful thrombectomy (TICI score of 0 ).

Although no statistical significance was noted, mean mRS at 3 months was 3 in each group. The 3-month mortality was similar in the two groups $(\mathrm{DA}=23.5 \%$ vs $\mathrm{TG}=25 \%)$.

Conclusion To the best of our knowledge, this is the first study reporting EVT data reflecting experiences from an academic program of an underserved state after the expansion of EVT time-window. This study showed no statistical difference in the outcomes of patients admitted directly through ED versus patients who were transferred from outside hospitals for EVT. Larger scale studies are needed to corroborate this data. Disclosures A. Zafar: None. S. Suriya: None. M. Farooqui: None. O. Owens: None. A. Alvarado Arias: None. A. Ikram: None. D. Sorte: None. A. Carlson: None. M. Torbey: None.

\section{E-118 MECHANICAL THROMBECTOMY IN DISTAL VESSELS: REVASCULARIZATION RATES, COMPLICATIONS AND FUNCTIONAL OUTCOME}

A Sweid*, J Head, S Tjoumakaris, V Xu, K Shivashankar, T Alexander, M Gooch, N Herial, R Rosenwasser, P Jabbour. Neurosurgery, Thomas Jefferson University, Philadelphia, PA

\subsection{6/neurintsurg-2019-SNIS. 193}

Background and importance Pivotal trials have shown the tremendous efficacy of mechanical thrombectomy in proximal occlusions (PO). However distal occlusions (DO) involving M2 and beyond, distal ACA (A2) and posterior cerebral arteries were not represented. The thoughts are that distal vessels supply a smaller brain area, however, with significant symptoms paired with accessible occlusions, the concept may need to be revisited. In this study, we investigated the efficacy and safety of distal circulation mechanical thrombectomies compared to proximal circulation mechanical thrombectomies.

Methods A retrospective review of patients presenting with distal circulation acute ischemic stroke (AIS) who underwent mechanical thrombectomy at a tertiary referral center between 2010 and 2018.

Results Out of 453 patients who underwent mechanical thrombectomy for AIS, 73 patients had a DO (average age 72 years, 59\% female). The mean NIHSS upon admission was 12 (95\% CI: 11-14). The mean procedure time was 45 minutes (95\% CI: 38-52) and mean time to revascularization, from symptom onset to finishing the thrombectomy procedure, was 522 minutes (95\% CI: 438-5607). 45\% ( $\mathrm{n}=34)$ of subjects received tPA. Vessels involved included M2 (89\%, $n=68)$, A2 $(3 \%, n=2)$, PCA $(3 \%, n=2)$, and M3/4 (5\%, n=4). A stentreiver alone was used in $11 \%$ of the cases $(n=8)$, aspiration alone in $8 \%(n=6)$, and both stentreiver and aspiration in $72 \%(\mathrm{n}=55)$, with failed attempts in $9 \%(\mathrm{n}=7)$ of cases. Most procedures required one pass to recanalize the vessel $(55 \%, n=42)$. TICI $\geq 2 \mathrm{~b}$ was achieved in $88 \%(n=67)$ of the subjects. Mean length of stay was eight days (95\% CI: 6-10). Mortality was noted in $8 \%(n=6)$ of patients and independent functional outcome of $\mathrm{mRS} \leq 2$ at three months was seen in $70.9 \%$ with clinical follow up. On univariate analysis peri-procedural distal emboli was significantly higher in DO group compared to PO group $(7 \%$ vs. $2 \%, \mathrm{p}<0.05)$ while post- 